\title{
Drell-Yan Lepton Pair Azimuthal Asymmetry in Hadronic Processes
}

\author{
Jian Zhou, ${ }^{1,2}$ Feng Yuan, ${ }^{2,3}$ and Zuo-Tang Liang ${ }^{1}$ \\ ${ }^{1}$ School of Physics, Shandong University, Jinan, Shandong 250100, China \\ ${ }^{2}$ Nuclear Science Division, Lawrence Berkeley National Laboratory, Berkeley, CA 94720 \\ ${ }^{3}$ RIKEN BNL Research Center, Building 510A, \\ Brookhaven National Laboratory, Upton, NY 11973
}

\begin{abstract}
We study the azimuthal asymmetry $(\cos 2 \phi)$ in the Drell-Yan lepton pair production in hadronic scattering processes at moderate transverse momentum region, taking into account the contributions from the twist-three quark-gluon correlations from the unpolarized hadrons. The contributions are found to dominate the asymmetry, and are not power suppressed by $q_{\perp} / Q$ at small $q_{\perp}$ where $q_{\perp}$ and $Q$ are the transverse momentum and invariant mass of the lepton pair. Accordingly, the Lam-Tung relation will be violated at this momentum region, and its violation depends on the twist-three functions. However, at large transverse momentum $q_{\perp} \sim Q$, the Lam-Tung relation still holds because all corrections are power suppressed by $\Lambda^{2} / q_{\perp}^{2} \sim \Lambda^{2} / Q^{2}$ where $\Lambda$ is the typical nonperturbative scale.
\end{abstract}


1. Introduction. Drell-Yan lepton pair production in hadronic scattering process [1] has been playing a very important role in studying nucleon structure and QCD dynamics [2], and is an important complementary to the deep inelastic scattering studies [3]. Moreover, the later development on the angular distribution of lepton pair has laid ground for parton model and QCD dynamics studies [4-6]. The lepton pair production in hadronic scattering,

$$
H_{1}+H_{2} \rightarrow \gamma^{*}+X \rightarrow \ell^{+} \ell^{-}+X
$$

comes from the virtual photon decays. At higher energies, we should also consider the weak boson $\left(Z^{0}\right)$ decay contributions. In this paper, we will limit our discussions only for the virtual photon decays. An extension to including $Z^{0}$ boson decay contributions is straightforward. In the leading order, virtual photon is produced through quark-antiquark annihilation process, $q \bar{q} \rightarrow \gamma^{*}$ in the parton picture [1]. In the rest frame of the lepton pair, we can define two angles [4]: one is the polar angle $\theta$ between one lepton momentum and the hadron; the azimuthal angle $\phi$ is defined as the angle between the hadronic plane and the lepton plane. Here and in the following discussions, we follow the Collins-Soper frame [4] to define these angles. Our results can be translated to other frames too. The general expression for the lepton pair angular distribution can be written as [4],

$$
\frac{d N}{d \Omega}=\left(1+\cos ^{2} \theta\right)+A_{0}\left(\frac{1}{2}-\frac{3}{2} \cos ^{2} \theta\right)+A_{1} \sin 2 \theta \cos \phi+\frac{A_{2}}{2} \sin ^{2} \theta \cos 2 \phi .
$$

It has been argued [4] that the coefficients $A_{0}, A_{1}$, and $A_{2}$ are all power suppressed at large $Q^{2}$, where $Q$ is the invariant mass of the lepton pair: $A_{0} \sim A_{2} \sim\left\langle k_{\perp}^{2}\right\rangle / Q^{2}$ and $A_{1} \sim\left\langle k_{\perp}\right\rangle / Q$ where $k_{\perp}$ is the typical transverse momentum scale in the process [4]. As a result, the lepton pair angular distribution will be dominated by $\left(1+\cos ^{2} \theta\right)$ in the small transverse momentum region. These power counting results were generalized to analyze the various relations between the above coefficients [5]. One of the interesting observations is the so-called Lam-Tung relation [5]: $2 \nu-(1-\lambda)=0$, where $\lambda=\left(2-3 A_{0}\right) /\left(2+A_{0}\right)$ and $\nu=2 A_{2} /\left(2+A_{0}\right)$. According to the above power counting results, this relation is obviously valid because $\lambda=1$ and $\nu=0$ at the leading power.

Finite transverse momentum of the lepton pair $\left(q_{\perp}\right)$ can be generated from gluon radiation from the leading partonic process, for example, through the quark-antiquark annihilation channel $q \bar{q} \rightarrow \gamma^{*} g$. Its contribution to the lepton pair angular distribution at finite transverse 
momentum was found [6],

$$
\frac{d N}{d \Omega}=\frac{3}{16 \pi}\left[\frac{Q^{2}+\frac{3}{2} q_{\perp}^{2}}{Q^{2}+q_{\perp}^{2}}+\frac{Q^{2}-\frac{1}{2} q_{\perp}^{2}}{Q^{2}+q_{\perp}^{2}} \cos ^{2} \theta+\frac{\frac{1}{2} q_{\perp}^{2}}{Q^{2}+q_{\perp}^{2}} \sin ^{2} \theta \cos 2 \phi+\ldots\right]
$$

where the $\sin 2 \theta$ term does not have simple expression and has been omitted in the above equation. Similar expression can be derived for the $q g \rightarrow \gamma^{*} q$ channel. Certainly, at finite transverse momentum, the angular coefficients $A_{i}$ in Eq. (2) are not zero any more. However, they do obey the power counting rule. For example, the $A_{2}$ coefficient in Eq. (2) from the contribution in Eq. (3) is power suppressed by $q_{\perp}^{2} / Q^{2}$ at low transverse momentum. However, the Lam-Tung relation is still valid for any value of $q_{\perp}$ from this contribution. This has been regarded as a simple prediction from QCD [6]. Higher order perturbative corrections to this relation has been calculated in the literature [7], where it was found that the violation is numerically very small. These studies have motivated many experimental investigations of the lepton pair angular distributions in hadronic scattering [8]. In particular, the $\pi$ induced fixed target experiment found large $\cos 2 \phi$ azimuthal asymmetry, which is difficult to understand $[8,9]$. The intrinsic transverse momentum effects have been proposed to explain these effects [10], which however is limited to small transverse momentum whereas the experimental data are in both small and moderate transverse momentum region.

Meanwhile, at low transverse momentum, there exist large logarithms in terms of $\alpha_{s}^{n} \ln ^{2 n}\left(Q^{2} / q_{\perp}^{2}\right)$ from the fixed order perturbative calculations [11-14]. Resummation of these larger logs has been formulated for the leading contribution from the partonic contribution, especially for the Drell-Yan lepton pair and vector boson production in hadronic scattering [14]. This corresponds to the leading term in the lepton pair angular distribution $\left(1+\cos ^{2} \theta\right)$ in Eq. (2). Because the rest terms $\left(A_{i}\right)$ are power suppressed in the limit $q_{\perp} / Q \ll 1$, it has been difficult to follow the Collins-Soper-Sterman resummation method [9]. Recently, there have been much efforts to study the soft gluon resummation for these higher order terms $[15,16]$, and hopefully these developments will lead to a final solution to this issue, especially following the original Collins-Soper-Sterman formalism.

In this paper, we study the lepton pair azimuthal asymmetry, in particular for the $A_{2}$ coefficient in Eq. (2) from different perspective. We are interested in its behavior at the moderate transverse momentum region $\Lambda_{Q C D} \ll q_{\perp} \ll Q$. At this region, there are two large momentum scales $q_{\perp}$ and $Q$. The contribution to $A_{2}$ from Eq. (3) is power suppressed by $q_{\perp}^{2} / Q^{2}$ as we mentioned above. However, from the following calculations we find that 
there exit contributions from the twist-three quark-gluon correlation functions from both incident hadrons, which are not power suppressed by $q_{\perp}^{2} / Q^{2}$, instead by $\Lambda^{2} / q_{\perp}^{2}$ where $\Lambda$ is the typical nonperturbative scale. These contribution will dominate $A_{2}$ coefficient at the moderate transverse momentum region, depending on the relative strength of $q_{\perp}^{2} / Q^{2}$ and $\Lambda^{2} / q_{\perp}^{2}{ }^{1}$. More importantly, because they are not power suppressed by $q_{\perp}^{2} / Q^{2}$, the soft gluon resummation can be performed following the classical Collins-Soper-Sterman approach, and the resummation effect will be similar to the leading term of $\left(1+\cos ^{2} \theta\right)$ (see for example, the similar study in [17]). We notice that another higher-twist effect from only one side of the incoming hadrons has also been studied in the literature [18, 19], which are different from our calculations below.

\section{Twist-three times twist-three contributions to the lepton pair azimuthal} asymmetry. From the general analysis of twist-three functions of the unpolarized hadrons [20, 21], we find the only twist-three function is $T_{F}^{(\sigma)}(x, y)$ which is equivalent to $E(x, y)$ studied in the literature $[21,22]$. It is defined as

$$
T_{F}^{(\sigma)}\left(x_{1}, x_{2}\right)=\int \frac{d y_{1}^{-} d y_{2}^{-}}{4 \pi} e^{i y_{2}^{-}\left(x_{2}-x_{1}\right) P^{+}+i y_{1}^{-} x_{1} P^{+}}\left\langle P\left|\bar{\psi}\left(0^{-}\right) \sigma^{+\mu} g F_{\mu}^{+}\left(y_{2}^{-}\right) \psi\left(y_{1}^{-}\right)\right| P\right\rangle,
$$

where $\mu$ is a transverse index, the sums over color and spin indices are implicit, $|P\rangle$ denotes the unpolarized hadron state with momentum $P=\left(P^{+}, 0^{-}, 0_{\perp}\right)$ and $P^{ \pm}=\left(P^{0} \pm P^{z}\right) / \sqrt{2}$, $\psi$ is the quark field, and $F_{+\mu}$ the gluon field tensor, and the gauge link has been suppressed. This correlation is a chiral-odd function, and can generate transverse polarized Hyperon production in unpolarized hadronic collisions [23]. Because of this chirality property, we have to introduce two correlation functions from both incoming hadrons.

To calculate its contribution, we follow the procedure outlined in [20, 24], and recent developments for the similar calculations [25-27]. In the collinear factorization framework, a general factorization formula for the contributions from the above correlation functions can be written as,

$$
\frac{d \sigma}{d^{4} q d \Omega}=\sum_{q} \int \frac{d x d x^{\prime}}{x} \frac{d z d z^{\prime}}{z} T_{F, q}^{(\sigma)}\left(x, x^{\prime}\right) T_{F, \bar{q}}^{(\sigma)}\left(z, z^{\prime}\right) \mathcal{H}\left(x, x^{\prime} ; z, z^{\prime} ; Q^{2}, q_{\perp}\right)
$$

\footnotetext{
${ }^{1}$ From power counting point of view, at this particular order, the new contribution will dominate in the region of $\Lambda^{2} \ll q_{\perp}^{2} \ll \Lambda Q$ whereas the contribution from Eq. (3) will dominate in the region of $\Lambda Q \ll q_{\perp}^{2} \ll Q^{2}$.
} 
where $T_{F, q}^{(\sigma)}\left(x, x^{\prime}\right)$ is the correlation function associated with the quark from hadron $H_{1}$ and $T_{F, \bar{q}}^{(\sigma)}\left(z, z^{\prime}\right)$ for the antiquark from hadron $H_{2}, \mathcal{H}$ is the hard part and can be calculated from perturbative partonic process. This factorization formula follows (as a conjecture) earlier general arguments for the higher-twist contributions to the hadronic cross sections [18, 20]. It will be very important to have a rigorous proof for this particular contribution as written in Eq. (5): the higher-twist effects coming from both sides of incoming hadrons, which is beyond the situations considered in [18]. Because of the higher-twist nature, it is always much more involved to calculate their contributions than those for the leading-twist contributions like Eq. (3). However, recent developments [25-27] have laid solid ground and useful technique to carry out those calculations. In particular, the two variables in $T_{F}^{(\sigma)}$ will be fixed by taking pole contributions, or equivalently by calculating the imaginary part of the interference of the scattering amplitudes. For example, in the above equation $x^{\prime}$ will be equal to $x$ depending on a soft or hard pole contribution [25-27]. In this paper, we will follow the procedure developed in [25-27] to calculate the hard part in Eq. (5).

First, we notice that the hard partonic part is separately gauge invariant summing up all possible diagrams. Therefore, we can carry out the calculations of these contributions with either $A^{+}$or $A_{\perp}$ field connecting the hard and soft parts. A particular example has been given in [26] for similar calculations. In our calculations, we find that it is more convenient to work the $A_{\perp}$ part, and construct the field tensor accordingly. However, it has been known that the individual diagrams associated with the $A_{\perp}$ field depend on the boundary condition, although the final results of all diagrams contributions do not $[28,30]$. Further calculations show that the retarded boundary condition $A_{\perp}\left(y^{-}=-\infty\right)=0$ will greatly simplify the derivations of Eq. (5). In the following, we will choose this boundary condition for the gauge field $A_{\perp}$ from both hadrons. Under this boundary condition, on the other hand, we have to take into account the contributions from the operator with partial derivative on the quark field $\left(\bar{\psi} \partial_{\perp} \psi\right)$. This is because, this matrix element can be related to the quark-gluon correlation function defined in Eq. (5),

$$
\int \frac{d y^{-}}{4 \pi} e^{i x P^{+} y^{-}}\left\langle P\left|\bar{\psi}(0) \sigma^{+\mu} i \partial_{\perp \mu} \psi_{\alpha}\left(y^{-}\right)\right| P\right\rangle=T_{F}^{(\sigma)}(x) \equiv T_{F}^{(\sigma)}(x, x),
$$

with retarded boundary condition for the gauge field [29]. Therefore, for a complete calculations, we have to calculate the diagrams associated with the operators $\left(\bar{\psi} \partial_{\perp} \psi\right)$, together with that of $\left(\bar{\psi} A_{\perp} \psi\right)[20]$. For the $A_{\perp}$ contribution, we can further deduce its contribution 

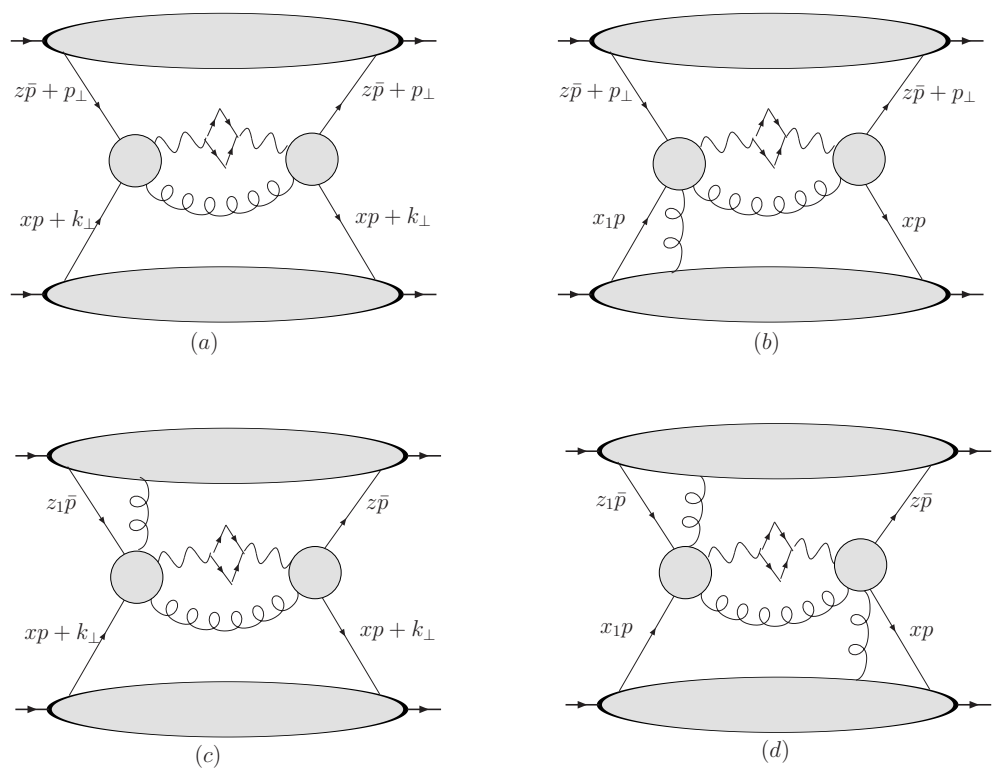

FIG. 1: Generic Feynman diagrams for the twist-three times twist-three contributions to the lepton pair azimuthal asymmetry in hadronic scattering process: (a) $\partial_{\perp}$ contribution from both sides of hadrons: (b-c) $\partial_{\perp}$ and $A_{\perp}$ from each side; (d) $A_{\perp}$ from both sides.

to that of $T_{F}^{(\sigma)}\left(x_{1} \cdot x_{2}\right)$. For example, under the same boundary condition for the gauge field $A_{\perp}$, we can write

$$
\begin{aligned}
& \int \frac{d y^{-} d y_{1}^{-}}{4 \pi} P^{+} e^{i x_{1} P^{+} y^{-}} e^{i\left(x-x_{1}\right) P^{+} y_{1}^{-}}\left\langle P S\left|\bar{\psi}\left(0^{-}\right) \sigma^{+\mu} g A_{\perp \mu}\left(y_{1}^{-}\right) \psi\left(y^{-}\right)\right| P S\right\rangle \\
= & \frac{i}{x-x_{1}+i \epsilon} T_{F}^{(\sigma)}\left(x, x_{1}\right)
\end{aligned}
$$

where the pole structure in the second line comes from the partial integral and the $i \epsilon$ prescription depends on the retarded boundary condition we are using. If we choose different boundary condition, this prescription shall change accordingly [29]. We have also checked that the above procedure can reproduce all previous results [25-27].

Following the above arguments, we plot the generic Feynman diagrams contributions in Fig. 1, where (a) is the contribution from $\partial_{\perp}$ operators from both sides of hadrons; (b-c) are those diagrams with $\partial_{\perp}$ and $A_{\perp}$ on either side; (d) for $A_{\perp}$ from both sides. There are four diagrams for Fig. 1(a), 12 diagrams for Fig. 1(b) and (c) respectively, and 208 diagrams for Fig. 1(d). As we mentioned above, in this paper we are interested in the cross section contributions in the moderate transverse momentum region $q_{\perp} \ll Q$. In carrying out these calculations, we will utilize the power counting method. We will only keep the leading power 
contributions and neglect all higher power corrections in terms of $q_{\perp} / Q$. The full expressions of our results will be presented in a separate publication. The advantage to use the retarded boundary condition is that we find that at the leading power, the contributions from the diagrams of Fig. 1(d) are either power suppressed or canceled out between soft and hard poles [29]. We are left with the contributions from Fig. 1(a-c) only, which are relatively easier to work out. In the limit of $q_{\perp} \ll Q$, the final result is

$$
\begin{aligned}
& \frac{d \sigma}{d^{4} q d \Omega}= \sigma_{0} \sin ^{2} \theta \cos (2 \phi) \frac{2}{q_{\perp}^{4}} \frac{\alpha_{s}}{2 \pi^{2}} \sum_{q} e_{q}^{2} \\
& \int \frac{d x}{x} \frac{d z}{z}\left\{A T_{F, \bar{q}}^{(\sigma)}(z, z) \delta(\hat{\xi}-1)+\bar{A} T_{F, q}^{(\sigma)}(x, x) \delta(\xi-1)\right. \\
&\left.\quad+2 C_{F} \delta(\xi-1) \delta(\hat{\xi}-1) T_{F, q}^{(\sigma)}(x, x) T_{F, \bar{q}}^{(\sigma)}(z, z) \ln \frac{Q^{2}}{q_{\perp}^{2}}\right\},
\end{aligned}
$$

where $\sigma_{0}=\alpha_{e m}^{2} / 6 S Q^{2}, \xi=x_{0} / x, \hat{\xi}=z_{0} / z$ with $x_{0}=\frac{Q}{\sqrt{S}} e^{y}$ and $z_{0}=\frac{Q}{\sqrt{S}} e^{-y}$, and $y$ is the rapidity of the lepton pair in the center of mass frame of incoming two hadrons and $S$ is the hadronic center of mass energy square. The coefficient $A$ is defined as

$$
A=\frac{1}{2 N_{c}}\left\{\left[x \frac{\partial}{\partial x} T_{F, q}^{(\sigma)}(x, x)\right] 2 \xi+T_{F, q}^{(\sigma)}(x, x) \frac{2 \xi(\xi-2)}{(1-\xi)_{+}}\right\}+\frac{C_{A}}{2} T_{F, q}^{(\sigma)}\left(x, x_{0}\right) \frac{2}{(1-\xi)_{+}},
$$

and similar expression holds for $\bar{A}$ in the above equation. These diagrams (Fig. 1) also contribute to other terms including $A_{0}$ and $A_{1}$ in Eq. (2), but they are all power suppressed by $q_{\perp}^{2} / Q^{2}$. From the above equations, we can see that this contribution to $A_{2}$ coefficient is not power suppressed by $q_{\perp}^{2} / Q^{2}$ at moderate transverse momentum region. It is the same order as the $\left(1+\cos ^{2} \theta\right)$ term in this power counting. Of course, it is suppressed by $\Lambda^{2} / q_{\perp}^{2}$ because of the higher-twist nature. This can also be seen from the above expression.

More importantly, the above result can also be reproduced by a transverse momentum dependent (TMD) factorization formalism [28, 30-35] with the so-called Boer-Mulders function from both hadrons $[36,37]$ at large transverse momentum which has been calculated in [23]. This demonstrates that in the intermediate transverse momentum region, for this part contribution, the twist-three times twist-three collinear factorization approach and the TMD factorization approach are consistent for the $\cos (2 \phi)$ azimuthal asymmetry in the unpolarized Drell-Yan processes. This is a nontrivial demonstration, because it goes beyond previous examples studied in the literature [27] where the twist-three effect from only one side of the incoming hadrons was considered. Because of this consistency, the energy 
evolution equation [13] (the Collins-Soper evolution equation) can be derived for this contribution, and the soft gluon resummation can be accordingly performed. This will significantly change the relative sizes of this contribution and the contribution from Eq. (3) to the $\cos 2 \phi$ asymmetry. We will leave a detailed study in a separate publication [29].

3. Conclusion. We have the following results for the angular distribution of the DrellYan lepton pair production in hadronic reactions,

- At moderate transverse momentum, $A_{2}$ is in order of $1, A_{0}$ is power suppressed by $q_{\perp}^{2} / Q^{2}$. As a result, the Lam-Tung relation will be violated because $\lambda$ is 1 whereas $\nu$ is order of 1 . Of course this violation will depend on the sizes of the twist-three correlation function $T_{F}^{(\sigma)}$ from both incoming hadrons. Furthermore, soft gluon resummation will not change the power counting result for $A_{2}$, because the leading order TMD factorization leads to the same resummation pattern similar to that discussed in [14], which is important to understand the angular distributions of the lepton pair at this momentum region [9].

- At large transverse momentum $q_{\perp} \sim Q$, however, both $A_{0}$ and $A_{2}$ are in order of 1 , and they are dominated by the contribution from the unpolarized quark and antiquark annihilation contribution Eq. (3). The contributions we calculated this paper are suppressed by $\Lambda^{2} / q_{\perp}^{2} \sim \Lambda^{2} / Q^{2}$. Because of this, the Lam-Tung relation will be valid again.

In summary, we have investigated the higher-twist effects to the Drell-Yan lepton pair angular distributions in hadron-hadron scattering processes. We found that the twist-three times twist-three contributions to the $\cos 2 \phi$ azimuthal asymmetry contribution are not power suppressed by $q_{\perp}^{2} / Q^{2}$, rather by $\Lambda^{2} / q_{\perp}^{2}$ at the moderate transverse momentum. We further argue that this part of contribution will not be affected by the soft gluon resummation effects, and the Lam-Tung relation will be modified at small and moderate transverse momentum. It will be interested to compare our predictions with the experimental data [8] and check the phenomenological importance of our results. It will also be interested to extend to other processes like the $\cos 2 \phi$ asymmetry in semi-inclusive hadron production in deep inelastic scattering and back-to-back two hadron production in $e^{+} e^{-}$annihilation processes where the similar effects shall play very important roles. 
We thank Jianwei Qiu and Werner Vogelsang for interesting discussions. This work was supported in part by the U.S. Department of Energy under contract DE-AC02-05CH11231 and the National Natural Science Foundation of China under the approval No. 10525523. We are grateful to RIKEN, Brookhaven National Laboratory and the U.S. Department of Energy (contract number DE-AC02-98CH10886) for providing the facilities essential for the completion of this work. J.Z. is partially supported by China Scholarship Council.

[1] S. D. Drell and T. M. Yan, Phys. Rev. Lett. 25, 316 (1970) [Erratum-ibid. 25, 902 (1970)].

[2] R. Brock et al. [CTEQ Collaboration], Rev. Mod. Phys. 67, 157 (1995).

[3] E. D. Bloom et al., Phys. Rev. Lett. 23, 930 (1969); M. Breidenbach et al., Phys. Rev. Lett. 23, 935 (1969).

[4] J. C. Collins and D. E. Soper, Phys. Rev. D 16, 2219 (1977).

[5] C. S. Lam and W. K. Tung, Phys. Rev. D 18, 2447 (1978); Phys. Lett. B 80, 228 (1979); Phys. Rev. D 21, 2712 (1980).

[6] J. C. Collins, Phys. Rev. Lett. 42, 291 (1979).

[7] E. Mirkes and J. Ohnemus, Phys. Rev. D 51, 4891 (1995).

[8] See for example, J. S. Conway et al., Phys. Rev. D 39, 92 (1989); L. Y. Zhu et al. [FNALE866/NuSea Collaboration], Phys. Rev. Lett. 99, 082301 (2007); arXiv:0811.4589 [nucl-ex].

[9] P. Chiappetta and M. Le Bellac, Z. Phys. C 32, 521 (1986).

[10] D. Boer, Phys. Rev. D 60, 014012 (1999).

[11] Y. L. Dokshitzer, D. Diakonov and S. I. Troian, Phys. Lett. B 78, 290 (1978); Phys. Rept. 58, 269 (1980).

[12] G. Parisi and R. Petronzio, Nucl. Phys. B 154, 427 (1979).

[13] J. C. Collins and D. E. Soper, Nucl. Phys. B 194, 445 (1982).

[14] J. C. Collins, D. E. Soper and G. Sterman, Nucl. Phys. B 250, 199 (1985).

[15] D. Boer and W. Vogelsang, Phys. Rev. D 74, 014004 (2006).

[16] E. L. Berger, J. W. Qiu and R. A. Rodriguez-Pedraza, Phys. Lett. B 656, 74 (2007); Phys. Rev. D 76, 074006 (2007).

[17] A. Idilbi, X. d. Ji, J. P. Ma and F. Yuan, Phys. Rev. D 70, 074021 (2004).

[18] J. W. Qiu and G. Sterman, Nucl. Phys. B 353, 105 (1991); Nucl. Phys. B 353, 137 (1991). 
[19] V. Braun, R. J. Fries, N. Mahnke and E. Stein, Nucl. Phys. B 589, 381 (2000) [Erratum-ibid. B 607, 433 (2001)].

[20] R. K. Ellis, W. Furmanski and R. Petronzio, Nucl. Phys. B 212, 29 (1983). R. K. Ellis, W. Furmanski and R. Petronzio, Nucl. Phys. B 207, 1 (1982).

[21] R. L. Jaffe and X. D. Ji, Nucl. Phys. B 375, 527 (1992).

[22] J. Zhou, F. Yuan and Z. T. Liang, arXiv:0812.4484 [hep-ph].

[23] J. Zhou, F. Yuan and Z. T. Liang, Phys. Rev. D 78, 114008 (2008).

[24] J.W. Qiu and G. Sterman, Phys. Rev. Lett. 67, 2264 (1991); Nucl. Phys. B 378, 52 (1992); Phys. Rev. D 59, 014004 (1998).

[25] C. Kouvaris, J. W. Qiu, W. Vogelsang and F. Yuan, Phys. Rev. D 74, 114013 (2006).

[26] H. Eguchi, Y. Koike and K. Tanaka, Nucl. Phys. B 752, 1 (2006); Nucl. Phys. B 763, 198 (2007).

[27] X. Ji, J. W. Qiu, W. Vogelsang and F. Yuan, Phys. Rev. Lett. 97, 082002 (2006); Phys. Rev. D 73, 094017 (2006); Phys. Lett. B 638, 178 (2006).

[28] X. Ji and F. Yuan, Phys. Lett. B 543, 66 (2002); A. V. Belitsky, X. Ji and F. Yuan, Nucl. Phys. B 656, 165 (2003).

[29] J. Zhou, F. Yuan, and Z. T. Liang, to be submitted.

[30] S. J. Brodsky, D. S. Hwang and I. Schmidt, Phys. Lett. B 530, 99 (2002); Nucl. Phys. B 642, $344(2002)$.

[31] J. C. Collins, Phys. Lett. B 536, 43 (2002).

[32] D. Boer, P. J. Mulders and F. Pijlman, Nucl. Phys. B 667, 201 (2003).

[33] X. Ji, J. P. Ma and F. Yuan, Phys. Rev. D 71, 034005 (2005); Phys. Lett. B 597, 299 (2004).

[34] J. C. Collins and A. Metz, Phys. Rev. Lett. 93, 252001 (2004).

[35] P. J. Mulders and R. D. Tangerman, Nucl. Phys. B 461, 197 (1996) [Erratum-ibid. B 484, $538(1997)]$.

[36] D. Boer and P. J. Mulders, Phys. Rev. D 57, 5780 (1998); D. Boer, S. J. Brodsky and D. S. Hwang, Phys. Rev. D 67, 054003 (2003).

[37] A. Metz, P. Schweitzer and T. Teckentrup, arXiv:0810.5212 [hep-ph]. 